\title{
Applying the code of medical ethics in the context of a pandemic: COVID-19 in Sri Lanka
}

${ }^{1}$ Karunatilaka KMAG

${ }^{1}$ DGH Mannar

\section{Abstract}

When thinking of something that would kill millions of people, a pandemic is the greatest risk. The outbreaks hit much harder to the lowerincome nations, given the lack of access to basic health services, health infrastructure, and effective infection control mechanisms. In this context, ethical aspects related to equity in resource allocation, selective vaccination, clinical trials involving human subjects, limitation of personal freedom such as quarantine regulations, and so forth need to be addressed by the healthcare professionals. Therefore, a recognized clinical ethics scheme is imperative to balance individual and population rights during the response to an outbreak.

\section{Key words}

clinical ethics, healthcare resource allocation, virus outbreak

\section{Introduction}

A pandemic is something that would surpass the death poll of past gigantic wars, devastate the world economy, and cause mass destruction to humanity. It brings out panic around the globe, but for a brief period. People forget easily: the leaders allocate more budget on the military than health care. However, the experts in the infectious disease field remain alert. In 2005, the WHO, who lead the global response to the pandemic, released the pandemic response blueprint (1). Similarly, in
September 2019, the WHO published 'A World at Risk', which predicts an imminent massive scale pandemic, where no country would be immune to the effects (2). Nevertheless, by the time they declared a pandemic on January 30, 2020, most of the countries' healthcare systems were not fully prepared. Hence, health care professionals are now faced with ethically challenging decisions in the background of the covid-19 pandemic and require guidance to articulate ethical principles for decision-making in health generally.

All the health care decisions are a balance between the basic principles; beneficence, non-maleficence, respect for autonomy, and justice. During a pandemic, it is of no difference. However, the way these principles are prioritized and balanced against each other ought to be adjusted so that some principles, which would be less relevant in ordinary days, are now highlighted (3). Moreover, medical professionals should weigh up their obligation towards patients, community, fellow professionals, and undeniably self.

\section{Responsibility towards the Patient- Resource allocation}

In a well-resourced health care system of a developed country, the principle of justice/ fairness appears to have little influence on day-to-day care. All the patients are offered the best possible care they need since the resources are adequate. Currently, however, the resources are scarce in proportion to the drastically rising number of 
Covid-19 patients. These resources include staff, space, and materials. Clinicians cannot provide the optimal care they would usually provide. On the other hand, the healthcare needs of a lowerresourced community would unquestionably overwhelm the resources available. This means that during a crisis, resource-allocation decisions are to depend heavily on the principle of justice all around the world (3).

Sri Lanka, being a developing south-Asian country with a wide range of races, cultures, and economic backgrounds, provides free health care to all. In case the pandemic worsens and the healthcare system falls into a crisis, the professionals are to follow a fair process when allocating resources and prioritizing patients. This proper resource allocation involves conserving the available, increasing further, prioritizing, and reinstating the resources. Equality should be maintained without being biased over personal characteristics. The people who have the greatest capacity to benefit and those who are needed for essential services should be prioritized $(3,4)$. Therefore, it is beneficial to have a formal criterion or a scoring system and be prepared while ensuring moral integrity.

\section{Responsibility towards community}

Clinical ethics also promotes empowering the health professionals' responsibilities towards the broader community. In the pre-pandemic context, the professionals' goal is to optimize the individual care of their patients. In contrast, now the clinical decisions aim at benefiting the whole community rather than one patient or currently identified patients (4). By the strategic use of justice, not only individual autonomy and beneficence but also nonmaleficence must be practiced across the whole population (3). For instance, although the clinicians are committed to patient-centered care, barring the visitors to a Covid-19 positive patient, which has a cost for the patient's well-being, is ethically justified concerning minimizing the spread of the virus to the community.

\section{Responsibility towards the other professionals and self}

The very next thought a healthcare professional struggles with is up to which extent he must risk the well-being of himself and his family to dispense his role-based responsibility to patient care. This is guided by two perspectives. One is the healthcare professionals' role-based obligation to expose themselves to a degree of risk and inconvenience to ensure optimal patient care (3). This is based on beneficence and non-maleficence, considering the patients' interests first. In usual circumstances, the system has the knowledge and resources to fulfill this commitment while protecting the staff. Health professionals would be using universal precautions with preventive protocols such as vaccination and prophylaxis. This is not the same as a pandemic. Hence, comes the second perspective, which is the society's responsibility to protect and compensate for the people who furnish their healthcare need (3).

The healthcare providers are also human beings: their well-being matters as everyone else's does. Furthermore, they are an essential part of providing patient care. Should there be a physical or mental inability for the staff to perform their duties, the patients, as well as other healthcare staff, are undoubtedly affected in return. Some features of the COVID-19 pandemic make it particularly difficult to protect the staff optimally: the virus is transmissible via air droplets and contaminated surfaces, a quick and reliable diagnostic tool is lacking, the supply of personal protective equipment (PPE) is limited. Consequently, it has become the responsibility of the leaders to initiate a structured approach to protect the health and safety of health staff. 


\section{Moral distress}

Another rarely discussed aspect of pandemic clinical ethics is moral distress. There is an intellectual difficulty for health professionals in making decisions as there are no distinct data or predictions about the disease dissimilar to a usual clinical scenario. Secondly, the value trade-offs that affect the people they care brings emotional difficulty. Subsequently, they feel that they are forced to take courses of action, which they feel are not right. The ideal way to overcome this situation is to first self-analyze the information considering the ethical fundamentals and discuss with colleagues to formulate a considered view. In this pandemic, clinicians are not to be left to make ethical decisions alone $(3,4)$.

The race does not end here. There are around 1.5 million viruses in wildlife that could leap to humans at any point. Human behavior as new mining, farming, and expanding into wildlife areas make pandemics inevitable. When it happens, there are no vaccines or counter-drugs, a global health emergency looms large. Therefore, humanity should be prepared and well-equipped with a legal, transparent, and educational basis to mitigate the effects of a pandemic in an ethically and morally justified approach.

\section{References}

1. World Health Organization. WHO checklist for influenza pandemic preparedness planning. Epidemic Alert \& Response. 2005; 1-39.

2. World Health Organization. A world at risk. Global Preparedness Monitoring Board. 2019. Available: https://apps.who.int/gpmb/assets/ annual_report/GPMB_annualreport_2019.pdf

3. Clinical Ethics and COVID-19-The University of Melbourne. [cited 24 Feb 2021]. Available: https://study.unimelb.edu.au/find/shortcourses/clinical-ethics-and-covid-19/

4. AMA Code of Medical Ethics: Guidance in a pandemic $\mid$ American Medical Association. [cited 24 Feb 2021]. Available: https://www. ama-assn.org/delivering-care/ethics/amacode-medical-ethics-guidance-pandemic 